\title{
Risks Associated With Conventional Humidifiers Adapted for High-Flow Nasal Cannula Therapy in Human Infants: Results of a Time and Motion Study
}

\author{
Robert Tero a, f, Joan Cecich ${ }^{\mathrm{b}}$, Omayra Sanabria ${ }^{\mathrm{c}}$, Shyan Sun ${ }^{\mathrm{a}}$, Jose Batista ${ }^{\mathrm{a}}$, Sara Stout ${ }^{\mathrm{b}}$, Danielle Zatt ${ }^{\mathrm{b}}$, \\ Robert Spoula $^{\mathrm{b}}$, Joan Gustafson ${ }^{\mathrm{b}}$, Sook Hee Lee ${ }^{\mathrm{c}}$, Thomas L. Miller ${ }^{\mathrm{d}}$ e
}

\begin{abstract}
Background: High-flow nasal cannula (HFNC) therapy was introduced into neonatology with novel heating-humidification technology; however, the therapy is currently being applied with adapted conventional humidifiers. Managing the rainout from these adapted systems is labor intensive and may be associated with water aspiration. A time and motion study was designed to evaluate the workflow needs and operational costs between a dedicated, integrated HFNC platform (Vapotherm Precision Flow; VT) and adapted conventional humidifier technology (Fisher \& Paykel MR850; FP).
\end{abstract}

Methods: Workflow was evaluated on HFNC devices in routine use. Observations were over 8 hours and staff self-reported device interactions. Workflow parameters included clearing condensate, need for suctioning, interface/tubing changes and charting. Device-related events that impacted workflow included the incidence of water in the airway, irritation and clinical sequelae. Data are reported as the mean number of contacts per device in an 8-hour shift.

Results: A total of 48 FP observations and 61 VT observations were collected across three sites. FP showed more therapist interventions $(4.5 \pm 1.5$ vs. $1.5 \pm 0.6 ; \mathrm{P}<0.001)$, and total unscheduled interventions $(1.1 \pm 1.6$ vs. $0.3 \pm 0.7 ; \mathrm{P}<0.001)$ compared to VT. Of the interventions, FP required draining tubing $2.1 \pm 1.0$ times vs. $0 \pm 0$ with VT $(\mathrm{P}<0.001)$. Rainout aspiration from the FP was associated with the $0.7 \pm 1.5$ device-related clinical events, versus $0 \pm 0$ events seen with VT $(\mathrm{P}<0.001)$.

Conclusions: HFNC using FP was associated with greater staff workload and patient risk related to the management of the rainout compared with VT. Thus, there may be an unaccounted cost beyond

Manuscript accepted for publication November 25, 2014

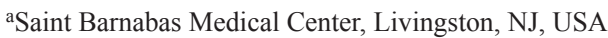

bDepartment of Respiratory Care, Edward Hospital, Naperville, IL, USA

c Jersey City Medical Center, Jersey City, NJ, USA

${ }^{d}$ Vapotherm, Inc., Exeter, NH, USA

eDepartment of Pediatrics, Jefferson Medical College, Philadelphia, PA, USA

${ }^{f}$ Corresponding Author: Robert Tero, Saint Barnabas Medical Center, 94 Old

Short Hills Road, Livingston, NJ 07039, USA.

Email: rtero@barnabashealth.org

doi: http://dx.doi.org/10.14740/ijcp172w circuit price with the use of conventional technologies for the administration of HFNC.

Keywords: High flow therapy; High-flow nasal cannula; Time and motion; Cost of care; Neonatology; Non-invasive respiratory care

\section{Introduction}

Heated, humidified high-flow nasal cannula (HFNC) therapy has been widely adopted over the past decade as non-invasive support for respiratory distress in neonatal intensive care units (NICUs) [1]. The operating premise is that by providing a flow of respiratory gas through a nasal cannula in excess of a patient's inspiratory flow demand, the patient will inhale the intended gas mixture without dilution via the entrainment of room air and provide for washout of nasopharyngeal dead space [2]. However, a critical technical aspect of HFNC is that the gas must be heated to body temperature and humidified to near saturation to avoid drying and injury to the nasal mucosa $[3,4]$ and subsequent infections [5].

The task of humidifying dry, cool respiratory gas to body temperature, and then delivering that gas to the patient without significant loss of energy and subsequent rainout is a challenge for humidification devices. Conventional technologies are prone to rainout [6-9], requiring staff to routinely disconnect and drain condensate from the circuitry. HFNC is a unique modality in that all of the gas travels through the cannula into the spontaneously breathing patient's nose, i.e. no circuit bias flow that bypasses the patient, making condensation management particularly important. Heated-humidified HFNC was first made clinically applicable in 2001 with the introduction of novel gas conditioning and delivery technology specifically for this purpose. These dedicated, integrated HFNC systems used a hollow-fiber membrane technology to condition gas precisely, and a water-jacketed delivery tube to eliminate rainout in the circuit. These HFNC-specific delivery tube and nasal cannula designs also incorporated a thinner internal diameter, smooth bore tubing to reduce transit time and turbulence in the flow path to further guard against rainout.

With the increasing popularity of HFNC and the clinical acceptance based on large efficacy trials [10-12], modifica- 
Table 1. Categories and Data Gathered on the Case Report Forms (CRF)

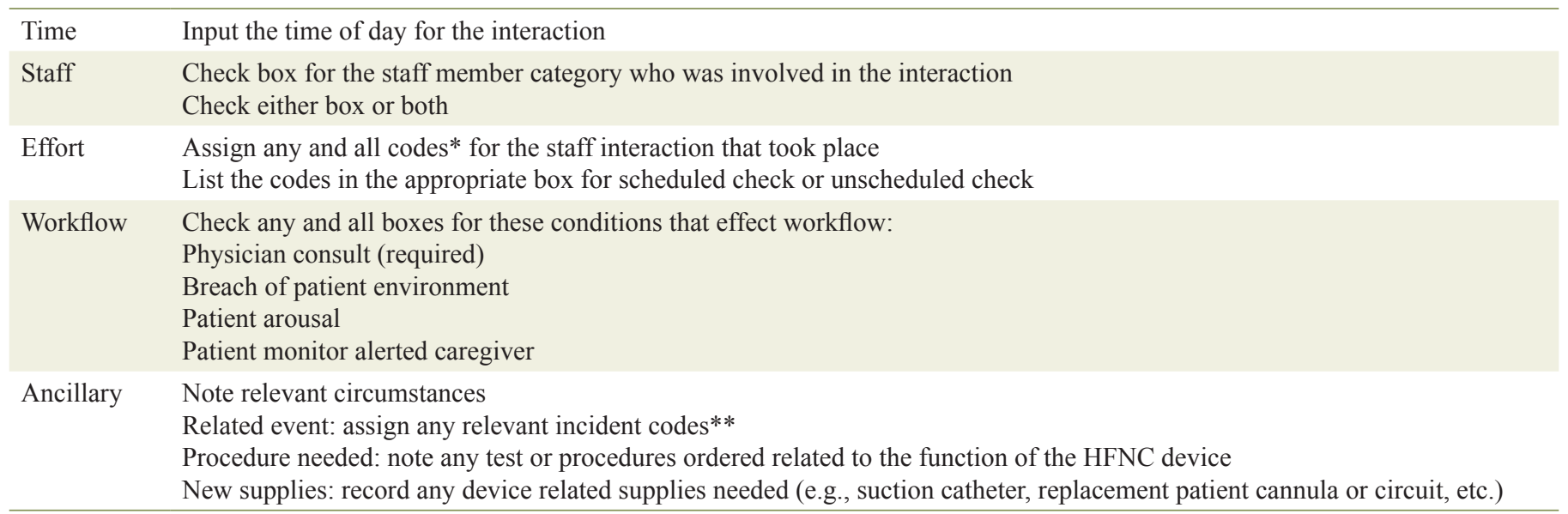

*Effort codes include: $(\mathrm{O})$ adjust patient setting: oxygen; $(\mathrm{T})$ adjust patient setting; temperature; $(\mathrm{F})$ adjust patient setting: flow; (W) clear condensed water; (S) suction the patient's airway; $(R)$ refit patient interface; $(C)$ change outpatient interface; $(B)$ change water bag; $(M)$ miscellaneous. ${ }^{* *}$ Incident codes include: (W) water in the airway; (D) pulse oximeter desaturation; (I) irritation/crying; (R) need for resuscitation; (B) bradycardic event; (A) apneic event; (M) miscellaneous.

tions of conventional technology have evolved into a lower cost approach using pass-over humidification and heated wire patient circuits where condensation buildup in the tubing is not unusual. Managing this rainout is thought to be labor intensive, and may be associated with device-related events that further impact staff time and workflow, and therefore additional costs of risk or condensation management may play a factor in the overall cost of care. The objective of this study was to conduct a time and motion analysis designed to evaluate the workflow needs and device-related events between a dedicated, integrated HFNC platform technology and an adapted conventional humidifier technology. The hypothesis was that compared to the purpose-built platform, the conventional technology would cost more to operate when considering the staff effort and other ancillary costs.

\section{Materials and Methods}

This time and motion study was modeled as a prospective, observational study of device-related caregiver effort, ancillary supplies/procedures and episodes of intervention associated with use of two HFNC device platforms for the delivery of non-invasive respiratory support in the NICU. Each observation dataset was gathered over an 8 -h period. The model was to include observations of a purpose-built HFNC platform device (VT; Precision Flow, Vapotherm, Exeter, NH, USA) and an adapted conventional humidifier technology (FP; MR850, Fisher \& Paykel Healthcare, Auckland, New Zealand), both of which are legally marketed and promoted with product features to support HFNC.

The study was conducted at three separate institutions: a 309-bed community hospital located in the Midwest, a 597-bed community/teaching hospital in the Northeast and a 300-bed community hospital in the Northeast. The Midwest center used both device platforms, first the FP and then the
VT following a period of acclimation, while the two Northeast hospitals, which are affiliated and regionally located used either FP or VT. Having one site (Midwest) sequentially use both devices was done to control for variability in policies and practice patterns among users. Conversely, having the affiliated and geographically similar centers (Northeast) each use one device, with which the respective staff was accustomed, controlled for the expertise in operating the respective device platform. Although this study was a device evaluation that did not include protected health information, the protocol was reviewed and approved by two Institutional Review Boards for the protection of human subjects.

\section{Subject selection criteria}

The subjects under study were the HFNC devices (being used in the NICU) not the patients, and no specific patient data were collected. Devices met the criteria for enrollment if being used to treat a patient who had been on the device for at least $24 \mathrm{~h}$ and by clinical judgment of the caregivers was not at risk for failure (re-intubation) within the projected observation period. Exclusion criteria consisted of a device being used on a patient who was unstable or medically complex. This strategy normalized the model to involve devices in use with patients who were in need of this level of respiratory support and were apparently stable, noting that all of these associated infants were in an intensive care setting.

\section{Study procedures}

To compare conditions under which devices were used, staff recorded the settings for cannula flow, gas temperature and percent oxygen in the delivered gas mixture at the beginning of the evaluation period. It was also indicated whether the de- 


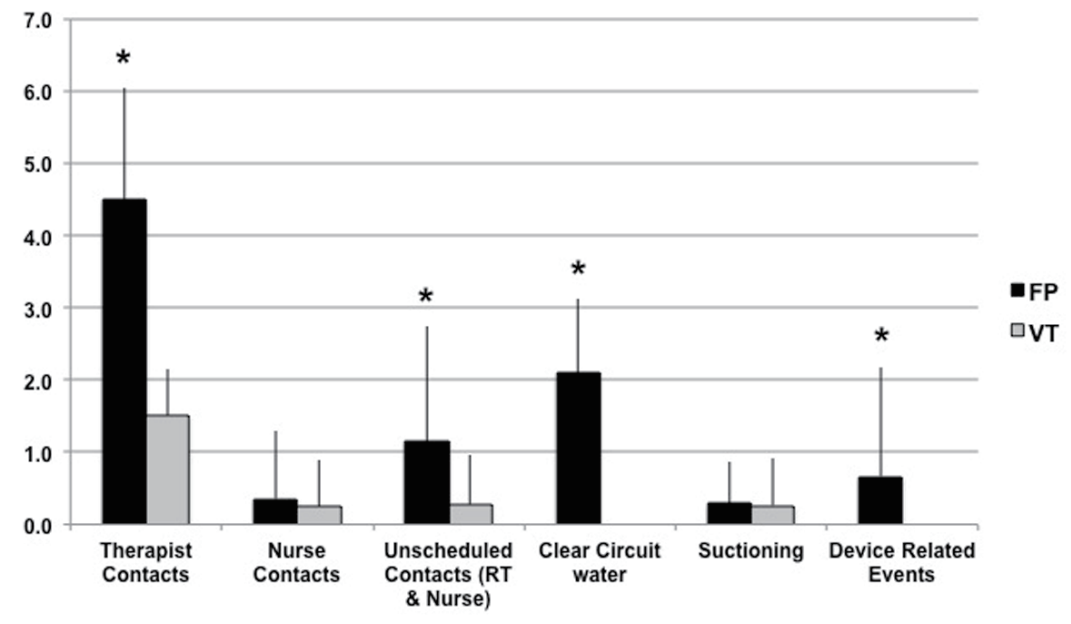

Figure 1. Comparison of primary effort-related categories and device-related outcomes. The tally of device-related staff interventions was compared between the conventional humidifier (FP) and a purpose built HFNC system (VT). The difference in device-associated staff contacts between platforms was significant for total respiratory therapist (RT) contacts as well as total unscheduled contacts (RT and nurse). Note that the total therapist contacts included routine checks/charting. A breakout of these contacts revealed differences in clearing circuit water, and during these contacts circuit condensate was associated with devicerelated events only in the FP platform. Data are presented as mean \pm SD per $8 \mathrm{~h}$ of observation. ${ }^{*} \mathrm{P}<0.001$.

vice was being used in conjunction with an isolette (regulated thermal environment) or an open bed. Approximately midway through the study, it was decided to record patient weight categories for the patients being treated to confirm similar clinical utilization between device platforms. These categories are: A (501 $\mathrm{g}$ to $1,000 \mathrm{~g}), \mathrm{B}(1,001 \mathrm{~g}$ to $1,500 \mathrm{~g}), \mathrm{C}(1,501 \mathrm{~g}$ to 2,000 $\mathrm{g})$, and $\mathrm{D}(\geq 2,001 \mathrm{~g})$.

Observations of the interactions with study subjects (i.e., devices) were recorded during the 8 -h periods using case report forms that were affixed to the device by the respiratory therapist on staff. All staff, including nurses and physicians as indicated, were instructed to list device-related interactions during the study period, i.e., workflow related to management of the device or a function of device technical performance. Staff were not to record workflow related to the patients' clinical needs that were not directly related to the performance of the HFNC device.

The clinical staff self-reported information about device interactions by adding tally marks on the case report forms for each intervention. Tallies of interventions were stratified by intervention category, staff classification and whether the intervention occurred during a scheduled check or an unscheduled response. As shown in Table 1, device-related interactions involved any action that directly manipulates the device, manipulates the patient interface, is a direct reaction to the device performance, and any time spent in consultation regarding the device function.

The primary effort and workflow parameters of interest included clearing condensate, patient suctioning, interface changes and charting. Importantly, this study was not intended to evaluate clinical outcomes, but rather device management needs. There was no recording of patient data; however, the occurrence of a clinical event that was associated with a device performance issue (e.g., aspiration of condensate) was to be noted by a tally mark. These clinical events included water in the airway, patient irritation, suctioning and the incidence of associated physiologic sequelae. Data on the incidence of various circumstances that affect workflow (e.g., physician consult needed, patient arousal, etc.) were tabulated for each condition, as well as conditions that reflect a direct cost (e.g., related test ordered, replacement disposable required, etc.).

\section{Data analysis}

Having no pre-existing data on variance from a study of this nature, sample size was cast for at least 30 observations in each device group. Each site was set to generate 30 observations for each device to allow for regional comparisons if warranted; however, provided that outcomes for each device group were consistent between the Midwest and respective Northeast centers, data could be pooled for a sample size of 60 observations in each device group.

Data for the number of interventions/events were compared between groups. Because the data are ordinal, recording the number of times an event occurs, it is not consistent with normality. However, non-parametric ranks procedures are compatible with this type of data. The two-sample KruskalWallis test was applicable for these results and tests the difference in the medians between the two groups. The results reported are two-sided tests at an alpha level of 0.05 and that the results are reported without adjustment for multiplicity.

\section{Results}

The Midwest center collected 17 observations with FP and 31 observations with VT. Northeast centers collected 31 observa- 


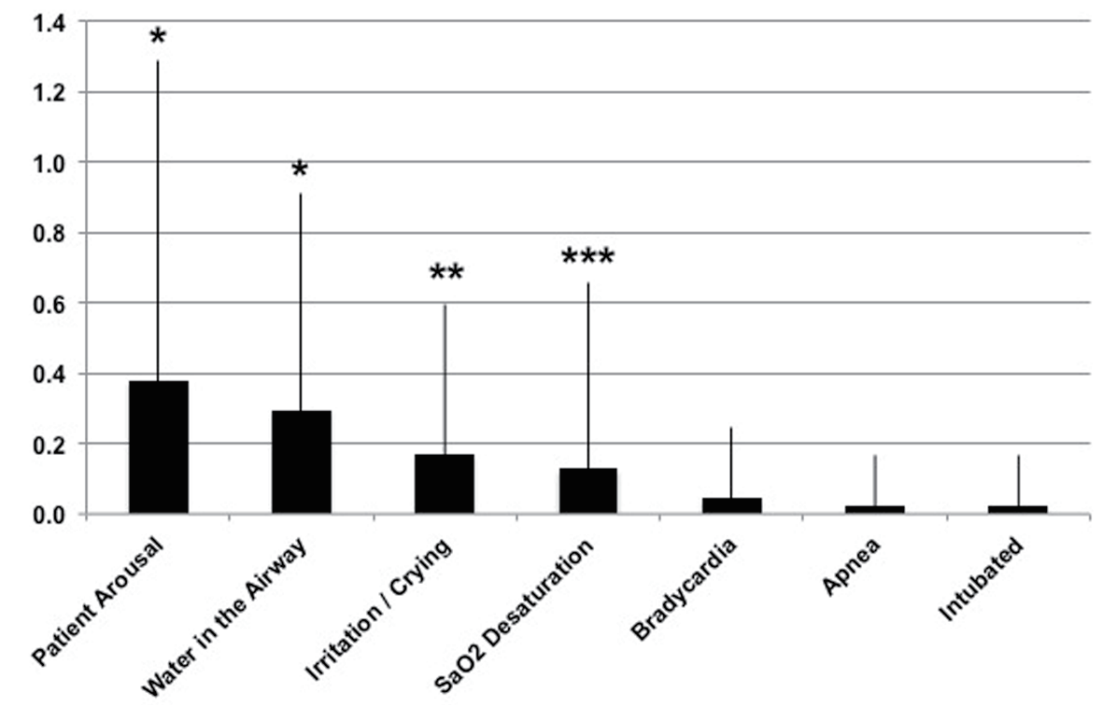

Figure 2. Breakout of device-related clinical events. Rainout aspiration, and in some cases other device related issues were associated with clinical events. In all cases the data for the purpose-built HFNC platform (Vapotherm) were $0 \pm 0$. The incidences of events with the conventional humidifier (Fisher \& Paykel) are shown here. Some of these values reached statistical significance compared to Vapotherm, but all have clinical relevance. Data are presented as mean \pm SD per $8 \mathrm{~h}$ of observation. ${ }^{*} \mathrm{P}<0.001$; ${ }^{* *} \mathrm{P}<0.01 ; \mathrm{P}<0.05$

tions with FP and 30 with VT. A decision was made for the Midwest center to halt data collection with the FP at 17 observations when routine auditing of the case report forms revealed the presence of clinical events related to rainout aspiration. The FP devices at the Midwest center appeared to accumulate rainout more rapidly. However, the staff workflow patterns were similar to the Northeast centers with each respective device, therefore the workflow data are pooled to give a total sample size of 48 observations with FP and 61 observations with VT.

There were differences between groups for the cannula flow rate $(2.8 \pm 1.2$ vs. $3.6 \pm 1.1 ; \mathrm{P}<0.001)$, but not temperature settings on the devices $(36.3 \pm 1.7$ vs. $35.3 \pm 1.9)$ and oxygen fraction delivered $(24.1 \pm 6.8 \%$ vs. $25.0 \pm 10.7 \%)$. Devices used in conjunction with an isolette represented $94 \%$ of the FP group and $77 \%$ of the VT group. The body weight categories for patients being treated were reported for 30 devices in the FP group (62\% of all cases) and 28 devices in the VT group ( $46 \%$ of all cases). The distribution showed that both device groups were used to treat infants across the spectrum of weight categories with FP and VT having, respectively, $10 \%$ vs. $21 \%$ in category A, $37 \%$ vs. $39 \%$ in B, $27 \%$ vs. $14 \%$ in $\mathrm{C}$ and $27 \%$ vs. $25 \%$ in D.

Figure 1 shows the comparison of primary effort related categories. Within the 8-h window of observation, FP showed more respiratory therapist interventions $(4.5 \pm 1.5$ vs. $1.5 \pm$ 0.6 ; $\mathrm{P}<0.001)$, and total unscheduled interventions by both therapist and nurses $(1.1 \pm 1.6$ vs. $0.3 \pm 0.7$; $\mathrm{P}<0.001)$ compared to VT. There was no difference in device-related nursing interventions $(0.4 \pm 0.9$ vs. $0.2 \pm 0.6)$. A breakout of the primary effort finding revealed underlying differences where FP had more incidences of draining rainout water from the patient tubing $(2.1 \pm 1.0$ times vs. $0 \pm 0 ; \mathrm{P}<0.001)$, which was not associated with more suctioning of the patient $(0.3 \pm 0.6$ vs.
$0.3 \pm 0.7)$

Also shown in Figure 1, FP resulted in $0.7 \pm 1.5$ device-related clinical events compared to $0 \pm 0$ events seen with VT (P $<0.001)$. These events were associated with rainout aspiration, and in some cases with other device functional issues. Associated with these events, there was a difference in the number of time a patient monitor or device alarm alerted the caregiver to a problem related to the device (FP $0.2 \pm 0.6$ vs. VT $0.0 \pm 0.0$; $\mathrm{P}=0.01)$.

Figure 2 shows a breakout of the clinical events seen with FP; note that in all cases the data for VT are $0 \pm 0$. Several comparisons reached significance between device platforms, which are the incidence of patient arousals $(\mathrm{FP}=0.4 \pm 0.9 ; \mathrm{P}$ $<0.001)$, water in the airway $(\mathrm{FP}=0.3 \pm 0.6 ; \mathrm{P}<0.001)$, crying/irritation of the baby $(\mathrm{FP}=0.2 \pm 0.4 ; \mathrm{P}<0.01)$ and arterial oxygen desaturation $(\mathrm{FP}=0.1 \pm 0.5 ; \mathrm{P}<0.05)$. Other noted device-related occurrences included re-intubation $(\mathrm{n}=1)$, bradycardia $(n=2)$ and apnea $(n=1)$. The device functional issues included three instances in the FP group where the temperature sensing mechanism faulted on the device, whereby the temperature probes required cleaning/replacing. This action is presumably a function of rainout water in the circuit affecting the temperature of the probes.

\section{Discussion}

This study was initiated as workflow analysis to determine if there was an incremental cost of time and effort needed to manage a conventional humidification platform adapted to the HFNC application which outweighed the lower material cost compared to the newer platform technology. A major impact of this study was that it revealed clinical risk associated with the 
management of rainout in the use of the adapted conventional technology. These data demonstrate that condensed water accumulating in the conventional circuitry is a real concern that requires a significant staff awareness and effort to mitigate this risk. Therefore, in choosing to use the adapted conventional technology over the purpose-built HFNC platform, administrators should consider the staffing and resource needs to manage this phenomenon weighted against the risk and potential cost of adverse events.

There was a difference in the way high flow therapy was utilized between groups, where the FP group used a lower mean flow rate of delivered gas. This could conceivably contribute to the rainout in this group because a lower flow allows the gas more time to cool as it travels through the circuit. However, several factors negate this comparison. First, the differences in mean values between groups for each of these comparisons were roughly one half of the respective group standard deviations. Therefore there was a greater range of settings within each group relative to the difference in means, whereas the outcomes within each group were extremely consistent; thus, the difference in means, albeit statistically significant, could not account for the differences in outcomes. Secondly, it should be considered that the use of a small bore tubing to reduce gas transit time in the circuit, as well as the ability to set a precise temperature, is among the features of the VT device platform which help to mitigate rainout.

There are several published studies that discuss the risks of rainout using conventional humidifier platforms in mechanically ventilated patients [6-9]. A number of these studies going back to the 1970s through the 2000s discuss these risks in the neonatal applications $[6,7,9]$. A set of articles published in Health Devices Journal in 2002 and 2005 discuss how the rainout from these conventional systems, including the Fisher \& Paykel MR850 used in this study, can cause ventilator systems to malfunction and/or shut down $[8,13]$. Aside from the risk of patient aspiration, rainout in the patient circuit presents a complex challenge for management. Rainout in the tubing is routinely dumped back into the reservoir to avoid discontinuation of therapy by breaking the circuit to drain. However, American Association for Respiratory Care clinical practice guidelines for humidification systems with invasive and noninvasive ventilation (2012) considers circuit condensation to be infectious waste (Section 12.3), which should "never be drained back into the humidifier reservoir" (Section 12.4) [14].

Rainout in the device circuit may be more of a hazard with HFNC compared to conventional ventilatory support systems. With conventional ventilatory and positive airway pressure circuitry, bias flow is used to generate pressure in the system wherein most of the gas flow in the circuit bypasses the patient's airway. With HFNC applications, all gas from the device is passed through the nasal cavity, thus the buildup of condensate should add more risk of aspiration. Subsequently, with the introduction of relatively significant volumes of water during spontaneous breathing, these infants may be more prone to altered breathing patterns which can either acutely, or by an additive effect, result in a significant clinical event.

As for the time and motion analysis, there was a significant and time-dependent workload burden on the care staff associated with the conventional humidifier. According to the current dataset, the therapists or nurses were required to clear water from the circuit tubing at least every $4 \mathrm{~h}(2.1 \pm 1.0$ instances of draining the tube per $8 \mathrm{~h}$ window) to keep the buildup of water under control. This equated to some unscheduled check, which would not only account for the time it takes to physically drain the fluid, but also the time to make additional rounds and interfere with other tasks that the staff may be responsible for. For example, if it took an average of $5 \mathrm{~min}$ to go to the bedside, dump the tubing condensate and assess the patient, the cost would be an extra $30 \mathrm{~min}$ of therapist time per day, per patient.

In addition, these data show that the rate of condensation buildup is dependent on extraneous factors, presumably environmental conditions such as room temperature and airflow patterns, which caused one of the centers to have episodes of rainout reaching the baby despite a similar number of tube drainings per shift. These unknown factors influencing rainout accumulation were associated with clinical events that may present another consideration of cost. For example, clinical events that were noted in the current study related to rainout aspiration were episodes of apnea and bradycardia. Apneic events complicated by bradycardia in preterm infants have been shown to be associated with decreased cerebral blood flow, which presents the risk of hypoxic-ischemic consequences to the brain [15]. Furthermore, the data from the current study associate rainout-related device function to an incident of reintubation for an infant receiving care. Moreover, the other clinical events shown to be associated with water aspiration herein may certainly lead to instability in this delicate patient population which lowers the threshold for a sentinel event.

\section{Limitations}

The devices were not randomly selected for inclusion in the observations. However, all devices should function to the manufacturers' specifications and therefore randomization should not be warranted. The self-report methodology has limitations with respect to accuracy of time spent for each device contact; however, this methodology was used in place of an observer model because of the challenges of controlling intra-observer error across three centers. Moreover, the model used here was not able to capture the time and effort spent outside of routine patient care for the planning and implementation of strategies to mitigate circuit rainout, such as localization of patient beds relative to air vents, etc.

\section{Conclusions}

In conclusion, as compared to the purpose-built platform, HFNC with an adapted conventional humidifier was associated with a significant increase in staff contacts and the potential for patient risk related to the management of rainout. Thus, there is an unaccounted cost beyond circuit price with the use of conventional technologies for the administration of HFNC. When using conventional humidification technology, consid- 
eration should be given to the cost of staffing and resources needed to manage circuit rainout and account the risk of clinical sequelae associated with condensate aspiration.

\section{Acknowledgement}

The authors would like to acknowledge Dr. Dmitry Dukuvny for his consultation in the design of the study model, as well as Dr. Richard Chiacchierini for his help with the statistical analysis.

\section{Funding Information}

This study was funded in part by Vapotherm, Inc., Exeter, NH and conducted at Saint Barnabas Medical Center, Edward Hospital and Jersey City Medical Center.

\section{Financial Disclosures}

Saint Barnabas Medical Center (Robert Tero, RRT as PI) and Jersey City Medical Center (Omayra Sanabria, RRT as PI) received research funding from Vapotherm for this study to cover IRB cost and PI effort allocation. Robert Tero is a moderator for an online forum supported by a grant from Vapotherm. Dr Miller is employed by Vapotherm.

\section{References}

1. Ward JJ. High-flow oxygen administration by nasal cannula for adult and perinatal patients. Respir Care. 2013;58(1):98-122.

2. Dysart K, Miller TL, Wolfson MR, Shaffer TH. Research in high flow therapy: mechanisms of action. Respir Med. 2009;103(10):1400-1405.

3. Williams R, Rankin N, Smith T, Galler D, Seakins P. Relationship between the humidity and temperature of inspired gas and the function of the airway mucosa. Crit
Care Med. 1996;24(11):1920-1929.

4. Kelly MG, McGarvey LP, Heaney LG, Elborn JS. Nasal septal perforation and oxygen cannulae. Hosp Med. 2001;62(4):248.

5. Kopelman AE, Holbert D. Use of oxygen cannulas in extremely low birthweight infants is associated with mucosal trauma and bleeding, and possibly with coagulase-negative staphylococcal sepsis. J Perinatol. 2003;23(2):94-97.

6. Ventilator rainout: patient care at odds with infectious waste control. Hosp Hazard Mater Manage. 1989;3(3):45 .

7. Rainout puts ventilator-dependent patients at risk. Health Devices. 2002;31(12):461-463.

8. Rainout from a Fisher \& Paykel heated humidification system can shut down certain ventilators. Health Devices. 2002;31(3):114-115.

9. Nelson D, McDonald JS. Heated humidification, temperature control, and "rainout" in neonatal ventilation. Respir Ther. 1977;7(2):41-44, 70-42.

10. Collins CL, Holberton JR, Barfield C, Davis PG. A randomized controlled trial to compare heated humidified high-flow nasal cannulae with nasal continuous positive airway pressure postextubation in premature infants. J Pediatr. 2013;162(5):949-954 e941.

11. Manley BJ, Owen LS, Doyle LW, Andersen CC, Cartwright DW, Pritchard MA, Donath SM, et al. High-flow nasal cannulae in very preterm infants after extubation. $\mathrm{N}$ Engl J Med. 2013;369(15):1425-1433.

12. Yoder BA, Stoddard RA, Li M, King J, Dirnberger DR, Abbasi S. Heated, humidified high-flow nasal cannula versus nasal CPAP for respiratory support in neonates. Pediatrics. 2013;131(5):e1482-1490.

13. Rainout from Fisher \& Paykel's 850 humidification system shuts down Respironics Esprit and adversely affects other ventilators. Health Devices. 2005;34(1):46-48.

14. Restrepo RD, Walsh BK. Humidification during invasive and noninvasive mechanical ventilation: 2012. Respir Care. 2012;57(5):782-788.

15. Perlman JM, Volpe JJ. Episodes of apnea and bradycardia in the preterm newborn: impact on cerebral circulation. Pediatrics. 1985;76(3):333-338. 